\title{
Predicting the Onset of Alcohol Use and the Development of Alcohol Use Disorder Among Indigenous Adolescents
}

\author{
Brian E. Armenta \\ University of Missouri
}

\author{
Kelley J. Sittner \\ Oklahoma State University
}

\author{
Les B. Whitbeck \\ University of Nebraska-Lincoln
}

\begin{abstract}
Empirical efforts to identify the predictors of drinking behavior among North American Indigenous adolescents are relatively limited. Using longitudinal data, this study considers perceived discrimination, positive drinker prototypes, and peer drinking behavior as risk factors for the onset of alcohol use and development of an alcohol use disorder among 674 Indigenous adolescents as they progressed from early to late adolescence $(M$ age at baseline $=11.11, S D=0.83$ ). Results showed that positive drinker prototypes and associations with peers who drink increased the risk for the onset of drinking, while perceived discrimination and associations with peers who drink increased the risk for the development of an alcohol use disorder. The theoretical and practical implications of our results are discussed.
\end{abstract}

For much of the 20th century research on alcohol use among North American Indigenous populations (i.e., Native American and Canadian First Nations people) was largely limited to reporting differences in drinking behaviors across ethnocultural groups. Although greater efforts have been made in recent years to identify the factors that influence alcohol use among North American Indigenous populations (e.g., Cheadle \& Whitbeck, 2011; Dickens, Dieterich, Henry, \& Beauvais, 2012; Swaim, Beauvais, Walker, \& Silk-Walker, 2011; Walls, Whitbeck, Hoyt, \& Johnson, 2007), this body of research remains relatively limited. The purpose of this study was to extend this body of research by considering the influence of perceived discrimination and positive drinker prototypes (i.e., positive beliefs about the typical adolescent drinker) on the development of drinking behaviors among a sample of North American Indigenous adolescents (hereafter referred to as Indigenous adolescents). We considered two drinking behaviors: the onset of alcohol use (i.e., the first time alcohol was used) and the development of an alcohol use disorder (i.e., alcohol abuse with or without dependence). We examined peer

The first author was partially supported by a grant from the National Institute of Alcohol Abuse and Alcoholism (AA013526; PI: Kenneth J. Sher) when writing this article.

Correspondence concerning this article should be addressed to Brian E. Armenta, Department of Psychological Sciences, University of Missouri, 200 S. 7th Street, Columbia, MO 65211. Electronic mail may be sent to armentab@missouri.edu. drinking behavior as an additional predictor in order to consider if perceived discrimination and positive drinker prototypes predict the onset of alcohol use and development of an alcohol use disorder after statistically controlling for this consistently strong and well-documented predictor adolescent drinking. Before describing our study, we briefly discuss the existing literature on positive drinker prototypes and perceived discrimination as risk factors for adolescent alcohol use, and further elaborate on our rationale for including peer drinking behavior in our analyses.

\section{Perceptions of Discrimination}

The belief that one has been discriminated against (i.e., perceived discrimination) has been conceptualized as a stressor (Clark, Anderson, Clark, \& Williams, 1999; Keyes, Hatzenbuehler, \& Hasin, 2011; Pascoe \& Smart Richman, 2009). As with other forms of stress, alcohol use has been discussed as a means of coping with perceived discrimination (Gerrard et al., 2012; Wei, Alvarez, Ku, Russell, \& Bonett, 2010; Wei, Heppner, Ku, \& Liao, 2010). Consistent with this view, cross-sectional and longitudinal studies have found that higher levels

(C) 2016 The Authors

Child Development (c) 2016 Society for Research in Child Development, Inc. All rights reserved. 0009-3920/2016/8703-0020

DOI: $10.1111 /$ cdev. 12506 
of perceived discrimination are associated with more frequent drinking and an increased risk for problematic drinking behavior among members of various ethnic, racial, and cultural minority groups (e.g., Williams \& Mohammed, 2009), including Indigenous adolescents (Cheadle \& Whitbeck, 2011; Whitbeck, Chen, Hoyt, \& Adams, 2004). We are aware of no studies, however, that have considered perceived discrimination in relation to the onset of alcohol use among adolescents from any ethnic, racial, or cultural minority group. When viewed as a coping mechanism, however, one may reasonably predict that perceived discrimination will increase the risk for the onset of alcohol use.

\section{Positive Drinker Prototypes}

Gibbons, Gerrard, Blanton, and Russell (1998; Gerrard, Gibbons, Houlihan, Stock, \& Pomery, 2008) argued that an adolescent's willingness to engage in a given risk behavior is partially influenced by the images that he or she holds about the prototypical adolescent who engages in that risk behavior (i.e., behavioral prototype). Ostensibly, when an adolescent engages in a given risk behavior, he- or she may (consciously or nonconsciously) adopt a view of him- or herself that is consistent with his or her view of the prototypical adolescent who engages in that risk behavior. From this perspective, an adolescent who views the prototypical adolescent drinker positively should be more willing to drink, as drinking would provide a means of viewing oneself more positively. In turn, given the appropriate social context (e.g., when at a party where alcohol is available), an adolescent who is more willing to drink should be at greater risk for drinking.

This argument reflects a social reaction view of adolescent drinking behavior, given that it describes adolescent drinking as occurring somewhat spontaneously in social situations that are conducive to drinking (Gibbons et al., 1998), rather than the result of a rational weighing of the costs and benefits involved in drinking (Ajzen, 1991; Fishbein, 1979). Importantly, a large body of research has demonstrated the utility of behavioral prototypes in predicting adolescent risk behaviors, either directly or indirectly via self-reported behavioral willingness or related constructs (see Gerrard et al., 2008, for a comprehensive review). Of specific relevance to the present study, our prior work (Armenta, Whitbeck, \& Hautala, 2015) has shown that more positive drinker prototypes were associated with more frequent drinking behavior among Indigenous early adolescents (i.e., ages $12-14$ years), both directly and indirectly via self-reported expectations that one will drink in the near future, which served as a proxy for willingness to drink (see Blanton, Gibbons, Gerrard, Conger, \& Smith, 1997). It is important to note that this study, which focused on the frequency of past year drinking behavior, was based on the same data used for the present study, which focuses on the onset of drinking behavior and the development of an alcohol use disorder.

We are aware of no studies that have considered the role of positive drinker prototypes in the onset of alcohol use among Indigenous or non-Indigenous adolescents. Gerrard, Gibbons, Stock, Vande Lune, and Cleveland (2005), however, showed that positive smoker prototypes were associated with the onset of smoking among a sample of African American preadolescents. This provides at least indirect evidence that positive drinker prototypes will be associated with the onset of drinking behavior. In contrast, we are aware of no direct or indirect evidence regarding the potential role of positive drinker prototypes in the development of an alcohol use disorder. Theoretically, positive drinker prototypes are described as influencing spontaneous uses of alcohol in situations that are conducive to drinking (Gerrard et al., 2008; Gibbons et al., 1998), rather than the sustained heavy drinking that is indicative of an alcohol use disorder. We nonetheless remained tentative regarding the association between positive drinker prototypes and the onset of an alcohol use disorder.

\section{Controlling for Peer Drinking Behavior}

Knowing whether perceived discrimination and positive drinker prototypes are linked to alcohol use is of theoretical interest and importance. The practical utility of knowing whether perceived discrimination and positive drinker prototypes are linked to alcohol use, however, requires one to take other key predictors into account. Scholars have long recognized that associating with peers who drink increases the risk for adolescent drinking behavior (e.g., Jessor \& Jessor, 1977). There are at least two potential reasons for this link (Borsari \& Carey, 2001). First, being around peers who drink may increase the risk for drinking simply due to greater exposure to alcohol. Second, being around peers who drink may lead to the belief that drinking is an acceptable, and perhaps even desirable or expected, behavior. Regardless of the specific reason(s), cross-sectional and longitudinal studies have shown that associating with peers who drink is one 
of the strongest and most consistent predictors of adolescent drinking behavior (Leung, Toumbourou, \& Hemphill, 2014), including the onset of alcohol use (e.g., Urberg, Değirmencioğlu, \& Pilgrim, 1997), frequency of alcohol use (e.g., Scholte, Poelen, Willemsen, Boomsma, \& Engels, 2008), amount of alcohol consumed (e.g., Bot, Engels, Knibbe, \& Meeus, 2005), and development of problematic alcohol use (e.g., Chassin, Pitts, \& Prost, 2002). Importantly, studies with Indigenous adolescents also have shown that associations with peers who engage in delinquent behaviors (including drinking) were positively associated with early onset of alcohol use (Boyd-Ball, Véronneau, Dishion, \& Kavanagh, 2014), past-month alcohol use (HeavyRunner-Rioux \& Hollist, 2010), and past-month binge drinking (Chen, Balan, \& Price, 2012).

Given the well-documented and strong effects of peer drinking behavior on adolescent alcohol use, we statistically controlled for peer drinking behavior in our analyses. We believe that this variable is especially important in considering the potential effects of positive drinker prototypes, as adolescents who associate with more peers who drink also may view the prototypical adolescent drinker more positively.

We recognize that adolescents who drink or who are inclined to drink may seek out peers who drink. Our reason for considering associations with peers who drink as a predictor of alcohol use, while not accounting for potential "selection effects," is twofold. First, associations with peers who drink are included in our analyses primarily for the sake of considering whether the predictive utility of perceived discrimination and positive drinker prototypes above and beyond this well-established predictor of alcohol use. Second, given our specific research questions, and the analytic model necessary to address those questions (i.e., discrete time survival model), considering the potential reciprocal association between the alcohol use outcomes and peer drinking behavior was not possible.

\section{Present Study}

Our goal was to extend the burgeoning body of research examining the antecedents of drinking behavior among Indigenous adolescents by considering perceived discrimination and positive drinker prototypes as risk factors for the onset of alcohol use and the development of an alcohol use disorder. We included peer drinking behavior as an additional predictor in order to verify that any observed associations were not accounted for by this consistently strong and well-documented predictor of adolescent alcohol use.

Because we were interested in predicting the first occurrence of the two drinking behaviors, we utilized discrete time series survival models to analyze our data. On the basis of previous research, we predicted that perceiving more discrimination, having more positive drinker prototypes, and associating with more peers who drink would increase the risk for the onset of alcohol use. We also predicted that perceiving more discrimination and associating with more peers who drink would increase the risk for the development of an alcohol use disorder. For reasons discussed earlier, however, we remained tentative regarding the potential role of positive drinker prototypes in the development of an alcohol use disorder.

\section{Method \\ Sample}

The data used for the study were drawn from an eight-wave longitudinal study (data collected annually) examining culture-specific risk and resilience factors among Indigenous adolescents in the U.S. Northern Midwest and Canada. The sample included 674 adolescents $\left(M_{\text {age }}=11.11, S D=0.83\right.$, $50.3 \%$ girls) at the first wave of the study. At Wave 1 , the primary caregivers reported an average annual per capita family income of $\$ 5,488$ $(S D=\$ 4,044)$, had an average of 4.35 children $(S D=2.05 ; \quad M$ children living at home $=2.56, S D=1.84$ ), and reported an average of 5.05 people $(S D=1.87)$ living in their household. In addition, $49.9 \%$ reported a gross annual household income below $\$ 25,000 ; 41.5 \%$ reported that they owned their home, $28.3 \%$ reported that they rented their home, 9.2\% reported living at home rent free, and $2.3 \%$ reported living with friends or family. Readers are referred to Whitbeck, Walls, and Sittner Hartshorn (2014) for additional sample information.

Of the Wave 1 sample, $94.4 \%$ completed Wave 2 (50\% girls), $92.9 \%$ completed Wave 3 (49.7\% girls), $87.2 \%$ completed Wave 4 (50.7\% girls), $89.8 \%$ completed Wave 5 (50.7\% girls), $87.7 \%$ completed Wave 6 (50.3\% girls), $84.4 \%$ completed Wave 7 (51\% girls), and $77.6 \%$ completed Wave 8 (52.8\% girls). Attempts to contact the entire baseline sample were made at each wave of the study; thus, participants were not dropped for missing one or more of the waves. Of the sample, $1.8 \%$ completed only one wave of the study, $1.5 \%$ completed only two waves 
(3.3\% completed two or fewer), $2.8 \%$ completed only three waves $(6.1 \%$ completed three or fewer), $2.1 \%$ completed only four waves $(8.2 \%$ completed four or fewer), $2.8 \%$ completed only five waves (11\% completed five or fewer), $6.7 \%$ completed only six waves $(17.7 \%$ completed six or fewer), $20.5 \%$ completed only seven waves $(38.2 \%$ completed seven or fewer), and $61.9 \%$ completed all of the waves. In addition, $1.8 \%$ dropped out of the study after completing Wave 1, 1\% after completing Wave 2, 2.1\% after completing Wave 3, 0.7\% after completing Wave $4,1.8 \%$ after completing Wave 5, $2.7 \%$ after completing Wave 6 , and $12.3 \%$ after completing Wave 7 , while $77.6 \%$ remained in the study until Wave 8. We describe our approach to handling the missing in a subsequent section.

\section{Study Design}

The study was designed in partnership with three Native American Reservations and five Canadian First Nation Reserves. As part of strict confidentiality agreements, the names of the cultural group and participating sites are not provided, nor were any attempts made to distinguish between participants from the various locations. Importantly, however, all participants share a common cultural tradition, identify as members of a single cultural group, and the same traditional language with only minor regional variations in dialects (all participants were fluent in English). At each site tribal advisory boards were responsible for advising the research team on questionnaire development and handling personnel issues. The interviewers and site coordinators all were approved by the advisory boards and were either enrolled tribal members or, in a very few cases, nonmember spouses of enrollees. The interviewers were trained prior to each wave of data collection concerning methodological guidelines for personal interviewing and protection of human subjects.

Prior to the first wave of data collection each participating reservation and reserve provided the research team with a list of all families with at least one tribally enrolled child between the ages of 10 and 12 years who lived on or near the reservation and reserve. An attempt to contact all families was made in an effort to obtain a representative sample of the target population. The families were formally recruited through home visits, during which they were presented with a traditional cultural gift and an overview of the project. For those families who agreed to participate $(79.4 \%$ of the contacted families), the target adolescent and at least one adult caretaker were interviewed once per year for 8 years. As compensation for their participation, the families received US $\$ 40$ per participant for each wave completed. The project was approved by and conducted in compliance with the reservation and reserve advisory boards and the institutional review board at the University of Nebraska-Lincoln.

\section{Measures}

There were variations in the measures that were administered to the participants across the eight waves of the study. The measure of positive drinker prototypes was administered only at Waves 2, 3, and 5, and the measures of perceived discrimination and peer drinking behavior were administered only at Waves $1-3,5$, and 7 . Diagnostic interviews, which included an assessment of alcohol use disorder, were administered at Waves $1,4,6$, and 8 , while the onset of alcohol use was assessed at all waves of the study. We ultimately restructured the data by age, which is discussed in detail in a subsequent section.

Perceived discrimination was assessed using an 11-item measure adapted from the Schedule of Racist Events (Landrine \& Klonoff, 1996). The items were modified to reflect perceived personal cultural discrimination, that is, perceptions that one has been discriminated against because of his or her membership in his or her (Indigenous) cultural group. This measure included items such as, "How often [in the past year] has a store owner, sales clerk, or person working at a place or business treated you in a disrespectful way because you are [a member of your cultural group]?" and "How often [in the past year] has someone threatened to harm you physically because you are [a member of your cultural group]?" Responses were provided on a 3-point scale, anchored by 1 (never) and 3 (many times). Mean scale scores were computed by averaging across the responses to the items. The alpha coefficients were $.79, .82, .79, .82$, and .84 for Waves $1,2,3,5$, and 7 , respectively.

Positive drinker prototypes were assessed using the approach developed by Gibbons et al. (1998). Participants were first asked to think about kids of their age who drink alcohol; they were instructed to think about their general image of kids who drink and not anyone in particular. They were then asked to indicate the degree to which they thought kids who drink are popular, smart, cool, tough, goodlooking, mature, dull or boring (reverse phrased), independent, and self-confident. Responses were provided on a 4-point scale, anchored by 1 (not at all) and 4 
(very). Mean scale scores were computed by averaging across the responses to the items, after reverse coding the single reverse phrased item. The alpha coefficients were .84, .86, and .87 for Waves 2, 3, and 5 , respectively.

Peer drinking behavior was assessed by asking participants how many of their three best friends drink alcohol. Responses were provided on a 4point scale, anchored by 0 (none) and 3 (all three).

Onset of alcohol use was assessed by asking participants if they had ever had a drink of beer, wine, or other kind of alcoholic beverage. They were advised that "sips don't count." Responses were coded as 0 (did not use alcohol at or before a given wave) and 1 (tried alcohol at or before a given wave).

Alcohol use disorder was assessed using the alcohol use disorder module from the adolescent self-report version of the Diagnostic Interview Schedule for Children Version IV (DISC-IV; Shaffer, Fisher, Lucas, Dulcan, \& Schwab-Stone, 2000). The standardized DISC-IV scoring algorithm was used to obtain diagnoses of lifetime alcohol abuse and dependence, as defined in the fourth edition of Diagnostic and Statistical Manual for Mental Disorders (DSM-IV; American Psychiatric Association, 2000). In order to be consistent with the fifth edition of the DSM (DSM-V; American Psychiatric Association, 2013), the alcohol abuse and dependence variables were combined into a single alcohol use disorder variable (i.e., alcohol abuse with or without dependence), which was coded as 0 (no alcohol use disorder at or before a given wave) and 1 (diagnosed with an alcohol use disorder at or before a given wave).

\section{Data Restructuring and Missing Data}

We restructured our data by age in order to consider potential developmental differences in the modeled associations (i.e., moderation by age). At Wave 1 , the adolescents were primarily $10(25.7 \%)$, $11(40.4 \%)$, or $12(30.7 \%)$ years old, but the sample also included two 9-year-olds and twenty 13-yearolds. To simplify the restructuring, the 9-year-olds were included in the 10-year-old group and the 13year-olds were included in the 12-year-old group. Analytically, restructuring the data by 1 -year age intervals (i.e., 11, 12, 13, 14, etc.) was not possible due to sparse data at some ages, which led to coverage issues in our analyses. We thus opted to restructure the data into the following age categories: 11-12, 13-14, 15-16, and 17-18. This resulted in a pattern of missing data that may be considered to be missing by design (i.e., due to the pattern of assessments administered; Graham, Taylor, Olchowski, \& Cumsille, 2006). In particular, for the adolescents who were 10 years old at Wave 1, data for alcohol use disorder were not available at ages 11-12 (corresponding to Waves 2 and 3) and data for positive drinker prototypes were not available at ages 15-16 (corresponding to Waves 6 and 7). In addition, for the adolescents who were 12 years old at Wave 1, data for positive drinker prototypes were not available at ages 11-12 (corresponding to Wave 1 for age 12; no data were collected at age 11 for these adolescents) and data for alcohol use disorder were not available at ages 13-14 (corresponding to Waves 2 and 3).

Data that are missing by design may be considered missing completely at random (MCAR; Little \& Rubin, 2002; Rubin, 1976), thus allowing for the estimation of unbiased parameter estimates using multiple imputation or maximum likelihood estimation (see Enders, 2010). A small portion of the data, however, was missing due to attrition or participant's nonresponse. We thus used the approach outlined by Little (1988) to test whether the pattern of missing data may be considered MCAR. The null hypothesis for this test is that a systematic pattern of bias does not exist as a result of the missing data; thus, a nonsignificant test value would indicate that the pattern of missingness may be treated as MCAR. We conducted this analysis using SPSS Version 20 (SPSS Inc., 2011). The test statistic was not statistically significant, $\chi^{2}(264)=291.74, p=.12$, suggesting that the pattern of missing data may be considered MCAR.

We used multiple imputation via SPSS Version 20 to account for the missing perceived discrimination, positive drinker prototypes, and peer drinking behavior data, and full information maximum likelihood estimation via Mplus Version 6.1 (Muthén \& Muthén, 1998-2011) to account for the missing onset of alcohol use and alcohol use disorder data. We did this because (a) Mplus does not account for missing predictor data (but does for missing outcome data) when using discrete time survival models (i.e., cases are listwise deleted) and (b) approaches to using multiple imputation with dichotomous variables are not well established (Enders, 2010). For the missing perceived discrimination, positive drinker prototypes, and peer drinking behavior data, we computed 100 multiple imputation data sets (Graham, Olchowski, \& Gilreath, 2007) using the Markov chain Monte Carlo algorithm. All additional analyses were conducted within Mplus using full information maximum likelihood estimation. 
The means and standard deviations for all of the study variables (averaged across the multiple imputation data sets) are provided in Table 1, as are the zero-order associations between the predictor variables and the alcohol use outcomes.

\section{Results}

To illustrate the patterns of alcohol use among our sample, we first estimated unconditional growth curve models to obtain the estimated change in the onset of alcohol use and alcohol use disorder across time. We then estimated unconditional hazard models to obtain the estimated hazard rates for the onset of alcohol use and alcohol use disorder across time. The hazard rates represent the number of adolescents who met criteria for an outcome (i.e., onset of alcohol use or alcohol use disorder) at a given age divided by the number of adolescents who had not met criteria for that outcome at or before that same age.

As can be seen in Figure 1, there was a steady increase in both alcohol use onset and alcohol use disorder from ages 11-12 to 17-18. An estimated 90.1\% of the adolescents had tried alcohol and an estimated $42.1 \%$ of the adolescents had met lifetime criteria for an alcohol use disorder by the time they were 1718 years old. We find it important to note that not all of the adolescents who tried alcohol continued drinking, which is not reflected in the figure. Rather, the alcohol use onset variable should be taken to indicate that an adolescent had tried alcohol at least one time. Also as shown in Figure 1, the hazard rate for alcohol use onset increased steadily from ages 11-12 to 13-14 to 17-18, while the hazard rate for alcohol use disorder increased from ages 11-12 to 13-14, but remained largely constant thereafter, 11-12 and 17-18.

For our primary analyses, we estimated separate discrete time survival models to predict the hazard functions for the two alcohol use outcomes. In Mplus, a discrete time survival model is estimated as a single-class latent variable mixture model, using the target outcome variable at each wave as observed indicators of a single latent factor, with the factor loadings and variance fixed to 1 and 0 , respectively (for full details, see Muthén \& Masyn, 2005). The latent variable represents the base hazard rate, or odds of ever meeting criteria for the target outcome, and may be predicted as a logistic function of one or more time-invariant covariates. The hazard functions at individual ages may further be predicted as a logistic function of one or more time-varying covariates.

To be comprehensive, we first estimated models for each of the predictor variables for each alcohol use outcome separately (individual predictor models). We then estimated models in which all of the predictors were included for each outcome separately (full models). For the models, perceived discrimination, positive drinker prototypes, and peer drinking behavior served as time-varying covariates. In order to control for potential gender differences in the alcohol use outcomes, we included

Table 1

Descriptive Statistics and Zero-Order Correlations Among the Study Variables

\begin{tabular}{|c|c|c|c|c|c|c|c|c|c|c|}
\hline & \multicolumn{2}{|c|}{ Ages 11-12 } & \multicolumn{2}{|c|}{ Ages 13-14 } & \multicolumn{2}{|c|}{ Ages 15-16 } & \multicolumn{2}{|c|}{ Ages 17-18 } & \multicolumn{2}{|c|}{ Descriptives } \\
\hline & Onset & Disorder & Onset & Disorder & Onset & Disorder & Onset & Disorder & $M$ & $S D$ \\
\hline \multicolumn{11}{|l|}{ Ages 11-12 } \\
\hline Peer drinking & $.27^{* *}$ & $.17^{* *}$ & $.22^{* *}$ & $.21^{* *}$ & $.18^{* *}$ & $.25^{* *}$ & $.14^{* *}$ & $.26^{* *}$ & 0.61 & 0.92 \\
\hline Perceived discrimination & $.13^{* *}$ & .06 & $.08^{*}$ & $.20^{* *}$ & $.09^{*}$ & $.14^{* *}$ & .07 & $.15^{* *}$ & 1.24 & 0.27 \\
\hline Positive drinker prototypes & .07 & .01 & $.17^{* *}$ & $.08^{*}$ & $.12^{* *}$ & $.12^{* *}$ & $.09 *$ & $.09^{*}$ & 1.83 & 0.59 \\
\hline \multicolumn{11}{|l|}{ Ages $13-14$} \\
\hline Peer drinking & $.21^{* *}$ & $.15^{* *}$ & $.25^{* *}$ & $.23^{* *}$ & $.25^{* *}$ & $.25^{* *}$ & $.14^{* *}$ & $.31^{* *}$ & 1.12 & 1.10 \\
\hline Perceived discrimination & $.13^{* *}$ & .04 & $.10^{* *}$ & $.17^{* *}$ & $.09^{*}$ & $.14^{* *}$ & .05 & $.16^{* *}$ & 1.21 & 0.26 \\
\hline Positive drinker prototypes & $.14^{* *}$ & $.09^{*}$ & $.16^{* *}$ & $.08^{*}$ & $.19^{* *}$ & $.12^{* *}$ & $.15^{* *}$ & $.20^{* *}$ & 2.01 & 0.61 \\
\hline \multicolumn{11}{|l|}{ Ages $15-16$} \\
\hline Peer drinking & $.12^{* *}$ & .07 & $.23^{* *}$ & $.14^{* *}$ & $.37^{* *}$ & $.25^{* *}$ & $.33^{* *}$ & $.33^{* *}$ & 1.83 & 1.06 \\
\hline Perceived discrimination & $.09^{*}$ & .05 & $.13^{* *}$ & $.19^{* *}$ & $.08^{*}$ & $.18^{* *}$ & $.09^{*}$ & $.18^{* *}$ & 1.21 & 0.26 \\
\hline Positive drinker prototypes & .07 & .02 & $.15^{* *}$ & .05 & $.11^{* *}$ & $.08^{*}$ & $.12^{* *}$ & $.10^{*}$ & 2.06 & 0.61 \\
\hline \multicolumn{11}{|l|}{ Descriptives } \\
\hline Percent & 25.8 & 2.2 & 62.2 & 17.5 & 81.6 & 30.7 & 90.9 & 42.1 & - & - \\
\hline
\end{tabular}

Note. Onset $=$ onset of alcohol use; disorder $=$ alcohol use disorder.

$* p \leq .05 . * * \leq .01$. 


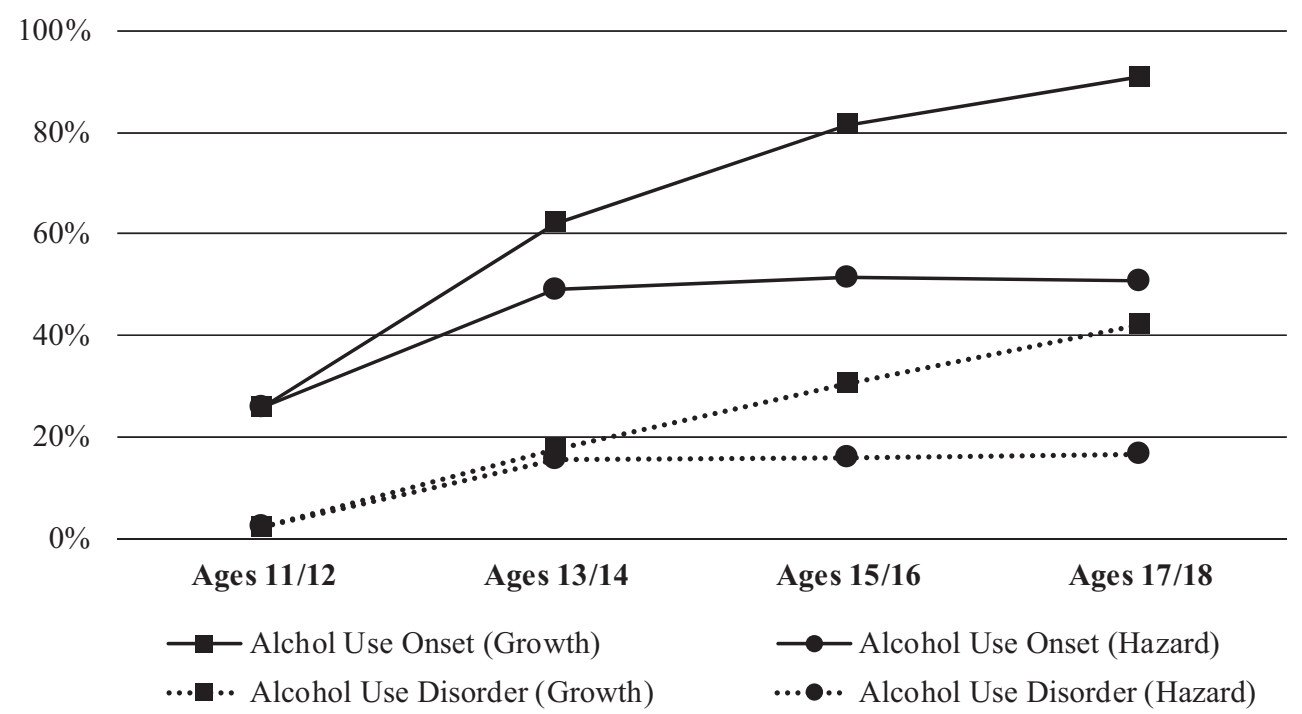

Figure 1. Estimated growth and hazard rates for alcohol use onset and alcohol use disorder.

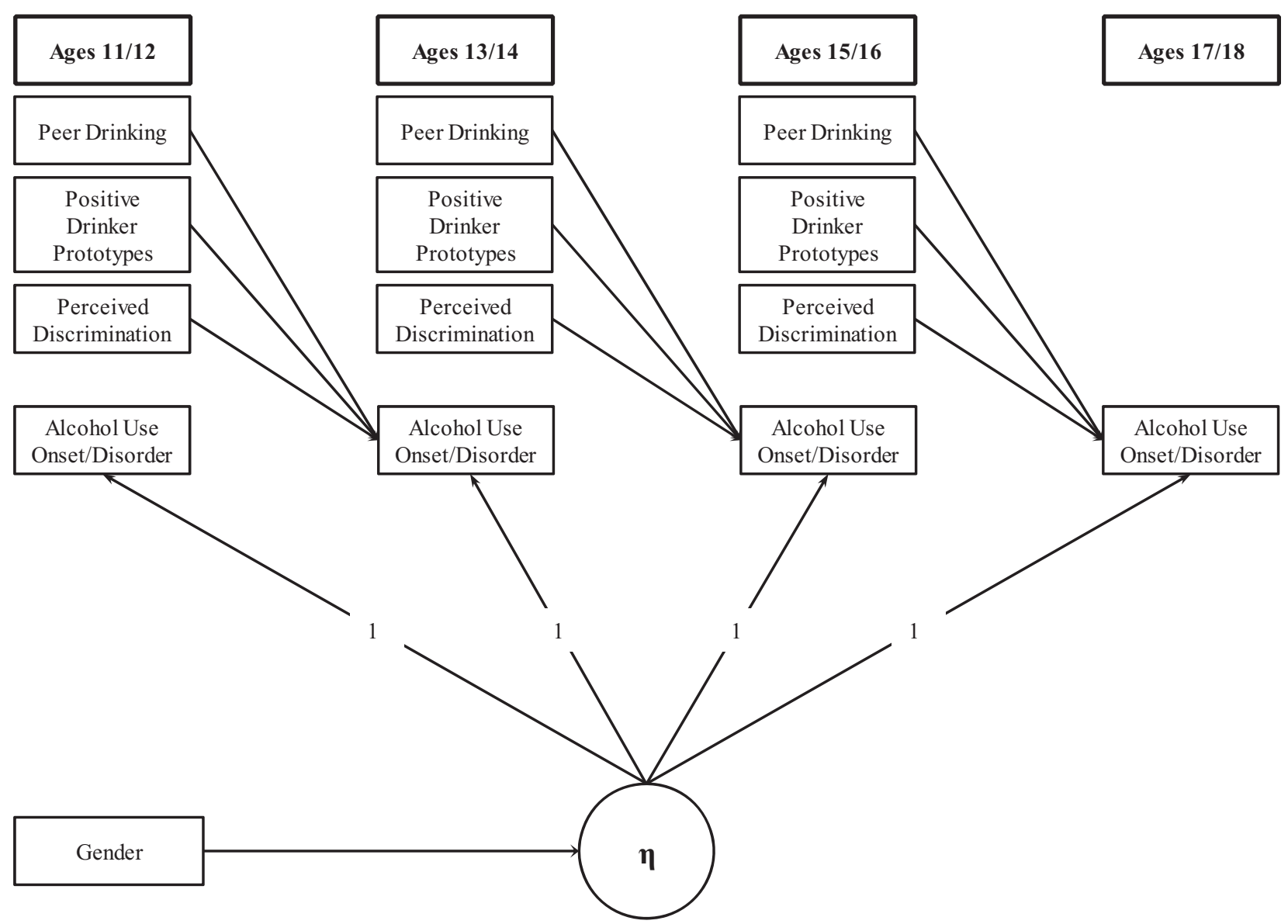

Figure 2. Final discrete time survival model.

gender as a time-invariant covariate (girls $=0$, boys $=1$ ). Our final analytic model is shown in Figure 2.
For the models with time-varying covariates, we estimated two separate models in order to test for potential age-based moderation effects. For the first 
model, the coefficients from the time-varying covariate(s) to the target outcome were allowed to estimate freely across time. For the second model, we constrained the path coefficients to be equivalent across time. We compared the fit of the unconstrained and constrained models by subtracting the log-likelihood value for the unconstrained model from that of the constrained model, which we then multiplied by $-2(-2 \Delta \mathrm{LL})$. The $-2 \Delta \mathrm{LL}$ value is chisquare distributed, with degrees of freedom equal to the difference in the number of parameters estimated for the unconstrained and constrained models. A significant test value would indicate a timebased moderation effect. A nonsignificant test value would indicate that there is no time-based moderation effect, which would allow us to report a single value for each predictor, as that value would hold similar across time.

Beginning with the individual predictor models, none of the constrained models resulted in a significant drop in model fit. For onset of alcohol use, the $-2 \Delta \mathrm{LL}$ values with $2 d f$ were $1.46(p=.48)$ for the perceived discrimination model, $.88(p=.64)$ for the positive drinker prototypes model, and 1.08 $(p=.58)$ for the peer drinking behavior model. For alcohol use disorder, the $-2 \Delta \mathrm{LL}$ values with $2 d f$ were $4.65(p=.10)$ for the perceived discrimination model, $1.05(p=.59)$ for the positive drinker prototypes model, and $3.82(p=.15)$ for the peer drinking model.

As shown in Table 2 (individual models), positive drinker prototypes and peer drinking behavior were positively and significantly related to the onset of alcohol use, while perceived discrimination, positive drinker prototypes, and peer drinking behavior were positively and significantly related to the development of an alcohol use disorder. Perceived discrimination was not significantly related to the onset of alcohol use and gender was not significantly related to either alcohol use outcome.

For the full models, the $-2 \Delta \mathrm{LL}$ values for the unconstrained versus constrained models with $6 \mathrm{df}$ were not statistically significant. The test values were $3.91(p=.69)$ for the alcohol use onset model and $11.60(p=.07)$ for the alcohol use disorder model. As shown in Table 2 (full models), the results followed the same pattern as the individual predictor models, with one exception. Specifically, the association between positive drinker prototypes and alcohol use disorder was not statistically significant.

It should be noted that we conducted secondary analyses in order to consider potential interaction effects for the three time-varying covariates. None of the two- or three-way interactions were statistically significant for alcohol use onset or alcohol use disorder (all $p \mathrm{~s}>.30$ ). Full results from these analyses are available upon request from the first author.

\section{Discussion}

In the present study, we examined perceived discrimination and positive drinker prototypes as risk factors for the onset of alcohol use and the develop-

\begin{tabular}{|c|c|c|c|c|c|c|c|c|c|c|}
\hline & \multicolumn{5}{|c|}{ Alcohol use onset } & \multicolumn{5}{|c|}{ Alcohol use disorder } \\
\hline & $b$ & $S E$ & $\beta$ & OR & $p$ & $b$ & $S E$ & $\beta$ & OR & $p$ \\
\hline \multicolumn{11}{|l|}{ Individual predictor models } \\
\hline \multicolumn{11}{|l|}{ Time-invariant covariates } \\
\hline Gender & .04 & .11 & .88 & 1.04 & .70 & 0.23 & .14 & 1.00 & 1.26 & .10 \\
\hline \multicolumn{11}{|l|}{ Time-varying covariates } \\
\hline Perceived discrimination & .48 & .33 & .07 & 1.62 & .15 & 1.23 & .28 & .17 & 3.42 & $<.01$ \\
\hline Positive drinker prototypes & .65 & .14 & .21 & 1.92 & $<.01$ & 0.44 & .15 & .14 & 1.55 & $<.01$ \\
\hline Peer drinking & .47 & .09 & .27 & 1.60 & $<.01$ & 0.46 & .07 & .26 & 1.58 & $<.01$ \\
\hline \multicolumn{11}{|l|}{ Full models } \\
\hline \multicolumn{11}{|l|}{ Time-invariant covariates } \\
\hline Gender & -.01 & .11 & .08 & 0.99 & .99 & 0.18 & .15 & .16 & 1.20 & .21 \\
\hline \multicolumn{11}{|l|}{ Time-varying covariates } \\
\hline Perceived discrimination & .22 & .35 & .03 & 1.25 & .52 & 0.96 & .29 & .12 & 2.61 & $<.01$ \\
\hline Positive drinker prototypes & .48 & .15 & .15 & 1.62 & $<.01$ & 0.11 & .17 & .03 & 1.12 & .50 \\
\hline Peer drinking & .38 & .09 & .21 & 1.46 & $<.01$ & 0.30 & .08 & .16 & 1.35 & $<.01$ \\
\hline
\end{tabular}

Note. $b=$ unstandardized path coefficient; $\beta=$ standardized path coefficient; $p=$ two-tailed probability value. 
ment of an alcohol use disorder (i.e., alcohol abuse with or without dependence) among a sample of Indigenous youth as they progressed from early (ages 11-12 years) to late (ages 17-18 years) adolescence. When considered as sole predictors, more positive drinker prototypes and associating with more peers who drink increased the risk for the onset of alcohol use. In addition, higher levels of perceived discrimination, more positive drinker prototypes, and associating with more peers who drink increased the risk for developing an alcohol use disorder. When considered as simultaneous predictors, the pattern of results remained similar, with one important exception: After statistically controlling for perceived discrimination, peer drinking, and gender, the effect of positive drinker prototypes on alcohol use disorder dropped to a nonsignificant level. Gender was not significantly associated with either alcohol use outcome, both when considered as a sole predictor and when considered simultaneously with the remaining predictor variables.

As noted in the Introduction, alcohol use is typically viewed as a means of coping with the negative experiences associated with perceived experiences with discrimination (Gerrard et al., 2012; Wei, Alvarez, et al., 2010; Wei, Heppner, et al., 2010). We thus predicted that adolescents who perceive more discrimination would be at greater risk for the onset of alcohol use and the development of an alcohol use disorder. Our results showed that perceptions of discrimination predicted risk for developing an alcohol use disorder, but not the onset of alcohol use. We are not exactly sure what to make of the latter finding and cannot speak firmly about it as our study represents the first attempt to examine perceived discrimination as a risk factor for the onset of alcohol use. It is possible, however, that the null effect is specific to our sample. Indeed, empirical evidence suggests that the onset of alcohol use occurs at an earlier age for Indigenous adolescents compared to their counterparts from other ethnocultural groups (Whitesell et al., 2012); this finding also was shown in the data used for the present article (Whitbeck \& Armenta, 2015). As such, it is possible that drinking is viewed as a more common behavior among Indigenous adolescents, potentially making normative social influences more central to the onset of drinking behavior. Further studies, however, will be necessary to consider this and other possible explanations for our results.

As discussed in more detail in the Introduction, positive drinker prototypes are believed to influence adolescent drinking behavior, which typically occurs in a spontaneous manner (e.g., when at a party where alcohol is available; Gerrard et al., 2008; Gibbons et al., 1998). Consistent with this suggestion, Gerrard et al. (2005) showed that positive smoker prototypes were positively associated with the onset of smoking among a sample of African American preadolescents. We thus predicted that positive drinker prototypes would increase the risk for the onset of alcohol use. Our results supported this prediction, thus providing an important replication of Gerrard et al.'s (2005) findings.

We remained tentative regarding the potential effect of positive drinker prototypes on the development of an alcohol use disorder. Empirically, we are aware of no studies that provide direct or indirect evidence to suggest that positive drinker prototypes should increase the risk for developing an alcohol use disorder. Theoretically, positive drinker prototypes are described as influencing spontaneous uses of alcohol in situations that are conducive to drinking (Gerrard et al., 2008; Gibbons et al., 1998). This does not directly suggest that positive drinker prototypes should influence the sustained, heavy drinking that is indicative of an alcohol use disorder.

When considered as a sole predictor of alcohol use disorder, adolescents who held more positive drinker prototypes appeared to be at greater risk for developing an alcohol use disorder. After taking perceived discrimination, peer drinking behavior, and gender into account, however, this association dropped to a level of nonsignificance. We conducted follow-up analyses to consider which of the additional predictors washed out the effect of positive drinker prototypes. To this end, we estimated separate models that included positive drinker prototypes and one additional predictor. Our analyses indicated that positive drinker prototypes became nonsignificant when peer drinking behavior, but not perceived discrimination or gender, was included as a second predictor (full results available upon request from the first author).

Knowing this pattern of results provides important information that scholars will need to take into consideration when considering the potential effects of positive drinker prototypes on adolescent alcohol use. In particular, as noted in the Introduction, we included peer drinking behavior in our analyses in order to control for this well-documented predictor of adolescent alcohol use. We also suggested that this was especially important for considering positive drinker prototypes, as adolescents who associate with more peers who drink also may have more positive drinker prototypes. Our results suggest 
that this is the case, although the exact reason for this is not clear. Specifically, our analytic approach does not allow us to consider if (a) adolescents who view the typical adolescent drinker more positive are more likely to seek out peers who drink, (b) adolescents who associate with more peers who drink develop more positive drinker prototypes, or (c) the overlap between positive drinker prototypes and peer drinking behavior is the result of some third variable. This is an empirical question that will require further consideration.

Our finding that associating with more peers who drink increases the risk for the onset of alcohol use and the development of an alcohol use disorder is consistent with a large body of research (e.g., Keyes, Hatzenbuehler, Grant, \& Hasin, 2012; Keyes et al., 2011). This includes studies conducted with Indigenous adolescents (Boyd-Ball et al., 2014; Chen et al., 2012; HeavyRunner-Rioux \& Hollist, 2010). These results, in isolation, are not novel, but nonetheless contribute additional evidence to support prior studies.

Two additional findings warrant mention. First, across all models considered, we did not find any age-based moderation effects. In other words, positive drinker prototypes and peer drinking behavior appear to exert a similar risk for the onset of alcohol use from early to late adolescence, and perceived discrimination and peer drinking behavior appear to exert a similar risk for the development of an alcohol use disorder from early to late adolescence.

Second, there were no gender differences in overall risk for alcohol use onset or the development of an alcohol use disorder. There is substantial evidence to suggest that adult men drink more than do adult women (see Nolen-Hoeksema \& Girgus, 1994). Among adolescents, when gender differences in alcohol use are identified, the pattern follows that of their adult counterparts (e.g., Deutsch, Steinley, \& Slutske, 2014). Findings regarding gender differences in alcohol use among adolescents, however, have been inconsistent, with several studies showing no gender differences (see Schulte, Ramo, \& Brown, 2009).

Importantly, in another article (Whitbeck \& Armenta, 2015), we found that the boys were more likely than the girls in our sample to have tried alcohol up to age 11, but this gender difference was not evident from ages 12 to 16 . We also found gender differences in the hazard rates for the onset of alcohol use, but only prior to age 11. Similarly, using data collected from a sample of Indigenous youths in middle school, Whitesell et al. (2012) found no significant gender difference in the hazard rate for the onset of alcohol use. Thus, at least among Indigenous adolescents, it appears that adolescent boys and girls may follow similar drinking patterns.

As with any study, our study is not without limitations, three of which we believe to be particularly noteworthy. First, we relied on adolescent selfreports of drinking behavior. Although our participants were informed that their responses would remain confidential, some of our participants may have either underreported (e.g., due to concerns regarding the information being shared with others or to avoid being judged negatively by the interviewer) or overreported (e.g., due to demand characteristics or an effort to appear more mature) their alcohol use. Future studies would benefit from obtaining information from multiple informants (e.g., peers, siblings, caretakers).

Second, although similar in some aspects, Indigenous groups differ in a number of ways (e.g., specific values and practices). Moreover, the experiences of Indigenous adolescents who do not live on or near their cultural group's reservation or reserve are likely to differ from their counterparts who do live on or near their cultural group's reservation or reserve (e.g., greater exposure to individuals from different cultures, fewer opportunities to engage in traditional cultural activities). We thus are not able to generalize our results to Indigenous adolescents from other cultural groups or nonreservation or reservation-residing Indigenous adolescents. Third, and related to the second limitation, we are unable to generalize our results to adolescents from other ethnocultural groups. As such, replications of our results with members of other Indigenous and nonIndigenous adolescent groups are needed in order to draw more confident conclusions regarding the links from positive drinker prototypes and perceived discrimination to the onset of alcohol use and the development of an alcohol use disorder among adolescents.

Despite these limitations, our results do highlight an important theoretical issue regarding positive drinker prototypes. As outlined by Gibbons et al. (1998; Gerrard et al., 2008), positive drinker prototypes are likely to influence spontaneous uses of alcohol (i.e., as exemplified by first use), but it is not fully clear if and why positive drinker prototypes should be related to the sustained heavy drinking that is indicative of an alcohol use disorder. In addition, it appears that sustained heavy drinking behavior may represent a means of coping with perceived experiences with discrimination. Perceptions of discrimination, however, appear to 
have no influence on the initiation of drinking. Along with the results for peer drinking behavior, this suggests that the initiation of alcohol use may be more heavily influenced by affiliations with peers who drink and perceptions of the prototypical adolescent drinker.

The results of our study also have potential implications for prevention and intervention efforts aimed at reducing (or at least delaying) the onset of alcohol use and the development of an alcohol use disorder among Indigenous adolescents (and perhaps adolescents from other ethnocultural groups). First, the onset of drinking and development of an alcohol use disorder may be altered by reducing adolescents' associations with peers who drink, perhaps through increases in parental or caregiver monitoring (BoydBall et al., 2014; Rodgers \& Fleming, 2003). Second, the initiation of drinking among Indigenous adolescents may be reduced (or at least delayed) through efforts to modify their beliefs about the prototypical adolescent who drinks, specifically, by decreasing their positive images of adolescents who drink, and perhaps increasing their positive images of adolescents who abstain from drinking (see Gerrard et al., 2008 , for a discussion of prevention and intervention efforts using this approach).

The possibility of reducing (or delaying) the development of an alcohol use disorder by reducing the amount of prejudice and discrimination that one is exposed to is an overly lofty aim. Recognizing, however, that the negative consequences stemming from experiences with discrimination result from how one processes his or her perceived experiences with discrimination opens up additional prevention and intervention options. For example, several studies suggest that socialization practices that teach children about being a member of a socially devalued group can have positive psychosocial consequences (see Hughes et al., 2006, for a review). What appear to be especially beneficial are messages that instill a sense of ethnocultural pride in children and adolescents. This finding makes sense given the large body of research demonstrating the psychosocial benefits of positively identifying with one's ethnocultural group (see Rivas-Drake et al., 2014, for a review). Research on ethnocultural identification (conceptualized as a psychological sense of group affirmation and belongingness) among Indigenous populations is limited. Nonetheless, there is some evidence that ethnocultural identification can buffer some of the negative psychosocial consequences of perceived discrimination among Indigenous adolescents (Galliher, Jones, \& Dahl, 2011). At this point, then, it would appear that socialization messages that instill a sense of ethnic pride in Indigenous adolescents may be useful for reducing the effects of perceived discrimination on the development of an alcohol use disorder.

To summarize, among our sample of Indigenous adolescents, we found that more positive drinker prototypes and associations with more friends who drink increased the risk for the onset of alcohol use, and higher levels of perceived discrimination and associations with more friends who drink increased the risk for the development of an alcohol use disorder. In our Discussion, we addressed the limitations to our study and highlighted the potential theoretical (i.e., regarding positive drinker prototypes) and practical (i.e., potential prevention and intervention efforts) implications of our results. Given the lack of published studies regarding the predictors of alcohol use behavior among Indigenous adolescents, these results make an important and substantial contribution to the literature. In closing, we encourage scholars to continue moving beyond making simple comparisons between the alcohol use of Indigenous and non-Indigenous groups and focus more heavily on the factors that predict alcohol use behavior among Indigenous populations. Such efforts are critical for scholars and practitioners who develop and implement prevention and intervention programs aimed at reducing (or delaying) the alcohol use among members of Indigenous groups.

\section{References}

Ajzen, I. (1991). The theory of planned behavior. Organizational Behavior and Human Decision Processes, 50, 179211. doi:10.1016/0749-5978(91)90020-T

American Psychiatric Association. (2000). Diagnostic and statistical manual of mental disorders (4th ed., text rev.). Washington, DC: Author.

American Psychiatric Association. (2013). Diagnostic and statistical manual of mental disorders (5th ed.). Washington, DC: Author.

Armenta, B. E., Whitbeck, L. B., \& Hautala, D. S. (2015). The utility of the prototype/willingness model in predicting alcohol use among North American Indigenous adolescents. Developmental Psychology, 51, 697-705. doi: $10.1037 / \mathrm{a} 0038978$

Blanton, H., Gibbons, F. X., Gerrard, M., Conger, K. J., \& Smith, G. E. (1997). Role of family and peers in the development of prototypes associated with substance use. Journal of Family Psychology, 11, 271-288. doi:10.1037/0893-3200.11.3.271

Borsari, B., \& Carey, K. B. (2001). Peer influences on college drinking: A review of the research. Journal of Sub- 
stance Abuse, 13, 391-424. doi:10.1016/S0899-3289(01) 00098-0

Bot, S. M., Engels, R. C. M. E., Knibbe, R. A., \& Meeus, W. H. J. (2005). Friend's drinking behaviour and adolescent alcohol consumption: The moderating role of friendship characteristics. Addictive Behaviors, 30, 929947. doi:10.1016/j.addbeh.2004.09.012

Boyd-Ball, A. J., Véronneau, M.-H., Dishion, T. J., \& Kavanagh, K. (2014). Monitoring and peer influences as predictors of increases in alcohol use among American Indian youth. Prevention Science, 15, 526-535. doi:10.1007/s11121-013-0399-1

Chassin, L., Pitts, S. C., \& Prost, J. (2002). Binge drinking trajectories from adolescence to emerging adulthood in a high-risk sample: Predictors and substance abuse outcomes. Journal of Consulting and Clinical Psychology, 70, 67-78. doi:10.1037/0022-006X.70.1.67

Cheadle, J. E., \& Whitbeck, L. B. (2011). Alcohol use trajectories and problem drinking over the course of adolescence: A study of North American indigenous youth and their caretakers. Journal of Health and Social Behavior, 52, 228-245. doi:10.1177/0022146510393973

Chen, H.-J., Balan, S., \& Price, R. K. (2012). Association of contextual factors with drug use and binge drinking among White, Native American, and mixed-race adolescents in the general population. Journal of Youth and Adolescence, 41, 1426-1441. doi:10.1007/s10964-012-9789-0

Clark, R., Anderson, N. B., Clark, V. R., \& Williams, D. R. (1999). Racism as a stressor for African Americans: A biopsychosocial model. American Psychologist, 54, 805-816. doi:10.1037/0003-066X.54.10.805

Deutsch, A. R., Steinley, D., \& Slutske, W. S. (2014). The role of gender and friends' gender on peer socialization of adolescent drinking: A prospective multilevel social network analysis. Journal of Youth and Adolescence, 43, 1421-1435. doi:10.1007/s10964-013-0048-9

Dickens, D. D., Dieterich, S. E., Henry, K. L., \& Beauvais, F. (2012). School bonding as a moderator of the effect of peer influences on alcohol among American Indian adolescents. Journal of Studies on Alcohol and Drugs, 73, 507-603. doi:10.15288/jsad.2012.73.597

Enders, C. K. (2010). Applied missing data analysis. New York, NY: Guilford.

Fishbein, M. (1979). A theory of reasoned action: Some applications and implications. Nebraska Symposium on Motivation, 27, 65-116.

Galliher, R. V., Jones, M. D., \& Dahl, A. (2011). Concurrent and longitudinal effects of ethnic identity and experiences of discrimination on psychosocial adjustment of Navajo adolescents. Developmental Psychology, 47, 509-526. doi:10.1037/a0021061

Gerrard, M., Gibbons, F. X., Houlihan, A. E., Stock, M. L., \& Pomery, E. A. (2008). A dual-process approach to health risk decision making: The prototype willingness model. Developmental Review, 28, 29-61. doi:10.1016/ j.dr.2007.10.001

Gerrard, M., Gibbons, F. X., Stock, M. L., Vande Lune, L. S., \& Cleveland, M. J. (2005). Images of smokers and willingness to smoke among African American preadolescents: An application of the prototype/willingness model of adolescent health risk behavior to smoking initiation. Journal of Pediatric Psychology, 30, 305318. doi:10.1093/jpepsy/jsi026

Gerrard, M., Stock, M. L., Roberts, M. E., Gibbons, F. X., O'Hara, R. E., Weng, C.-Y., \& Wills, T. A. (2012). Coping with racial discrimination: The role of substance use. Psychology of Addictive Behaviors, 26, 550-560. doi:10.1037/a0027711

Gibbons, F. X., Gerrard, M., Blanton, H., \& Russell, D. W. (1998). Reasoned action and social reaction: Willingness and intention as independent predictors of health risk. Journal of Personality and Social Psychology, 74, 11641180. doi:10.1037/0022-3514.74.5.1164

Graham, J. W., Olchowski, A. E., \& Gilreath, T. D. (2007). How many imputations are really needed? Some practical clarifications of multiple imputation theory. Prevention Science, 8, 206-213. doi:10.1007/s11121-0070070-9

Graham, J. W., Taylor, B. J., Olchowski, A. E., \& Cumsille, P. E. (2006). Planned missing data designs in psychological research. Psychological Methods, 11, 323-343. doi:10.1037/1082-989X.11.4.323

HeavyRunner-Rioux, A. R., \& Hollist, D. R. (2010). Community, family, and peer influences on alcohol, marijuana, and illicit drug use among a sample of Native American youth: An analysis of predictive factors. Journal of Ethnicity in Substance Abuse, 9, 260-283. doi:10.1080/15332640.2010.522893

Hughes, D., Rodriguez, J., Smith, E. P., Johnson, D. J., Stevenson, H. C., \& Spicer, P. (2006). Parents' ethnicracial socialization practices: A review of research and directions for future study. Developmental Psychology, 42, 747-770. doi:10.1037/0012-1649.42.5.747

Jessor, R., \& Jessor, S. L. (1977). Problem behavior and psychosocial development: A longitudinal study of youth. New York, NY: Academic Press.

Keyes, K. M., Hatzenbuehler, M. L., Grant, B. F., \& Hasin, D. S. (2012). Stress and alcohol: Epidemiologic evidence. Alcohol Research: Current Reviews, 34, 391-400.

Keyes, K. M., Hatzenbuehler, M. L., \& Hasin, D. S. (2011). Stressful life experiences, alcohol consumption, and alcohol use disorders: The epidemiologic evidence for four main types of stressors. Psychopharmacology, 218, 1-17. doi:10.1007/s00213-011-2236-1

Landrine, H., \& Klonoff, E. A. (1996). The Schedule of Racist Events: A measure of racial discrimination and a study of its negative physical and mental health consequences. Journal of Black Psychology, 22, 144-168. doi:10.1177/00957984960222002

Leung, R. K., Toumbourou, J. W., \& Hemphill, S. A. (2014). The effect of peer influence and selection processes on adolescent alcohol use: A systematic review of longitudinal studies. Health Psychology Review, 8, 426-457. doi:10.1080/17437199.2011.587961

Little, R. J. A. (1988). A test of missing completely at random for multivariate data with missing values. Journal 
of the American Statistical Association, 83, 1198-1202. doi: $10.2307 / 2290157$

Little, R. J. A., \& Rubin, D. B. (2002). Statistical analysis with missing data. Hoboken, NJ: Wiley.

Muthén, B., \& Masyn, K. (2005). Discrete-time survival mixture analysis. Journal of Educational and Behavioral Statistics, 30, 27-58. doi:10.3102/10769986030001027

Muthén, L. K., \& Muthén, B. O. (1998-2011). Mplus user's guide. Los Angeles, CA: Author.

Nolen-Hoeksema, S., \& Girgus, J. S. (1994). The emergence of gender differences in depression during adolescence. Psychological Bulletin, 115, 424-443. doi:10.1037/0033-2909.115.3.424

Pascoe, E. A., \& Smart Richman, L. (2009). Perceived discrimination and health: A meta-analytic review. Psychological Bulletin, 135, 531-554. doi:10.1037/a0016059

Rivas-Drake, D., Seaton, E. K., Markstrom, C., Quintana, S., Syed, M., Lee, R. M., . . . Yip, T. (2014). Ethnic and racial identity in adolescence: Implications for psychosocial, academic, and health outcomes. Child Development, 85, 40-57. doi:10.1111/cdev.12200

Rodgers, K. B., \& Fleming, W. M. (2003). Individual, family, and community factors related to alcohol use among Native American adolescents. Adolescent E Family Health, 3, 140-147.

Rubin, D. B. (1976). Inference and missing data. Biometrika, 63, 581-592. doi:10.1093/biomet/63.3.581

Scholte, R. H. J., Poelen, E. A. P., Willemsen, G., Boomsma, D. I., \& Engels, R. C. M. E. (2008). Relative risks of adolescent and young adult alcohol use: The role of drinking fathers, mothers, siblings, and friends. Addictive Behaviors, 33, 1-14. doi:10.1016/j.addbeh.2007.04.015

Schulte, M. T., Ramo, D., \& Brown, S. A. (2009). Gender differences in factors influencing alcohol use and drinking progression among adolescents. Clinical Psychology Review, 29, 535-547. doi:10.1016/j.cpr.2009.06.003

Shaffer, D., Fisher, P., Lucas, C. P., Dulcan, M. K., \& Schwab-Stone, M. E. (2000). NIMH Diagnostic Interview Schedule for Children Version IV (NIMH DISC-IV): Description, differences from previous versions, and reliability of some common diagnoses. Journal of the American Academy of Child $\mathcal{E}$ Adolescent Psychiatry, 39, 28-38. doi:10.1097/00004583-200001000-00014

SPSS Inc. (2011). SPSS for Windows (Version 20) [Computer software]. Chicago, IL: Author.

Swaim, R. C., Beauvais, F., Walker, R. D., \& Silk-Walker, P. (2011). The effects of parental diagnosis and changing family norms on alcohol use and related problems among urban American Indian adolescents. American Journal on Addictions, 20, 212-219. doi:10.1111/j.1521-03 91.2011.00129.x

Urberg, K. A., Değirmencioğlu, S. M., \& Pilgrim, C. (1997). Close friend and group influence on adolescent cigarette smoking and alcohol use. Developmental Psychology, 33, 834-844. doi:10.1037/0012-1649.33.5.834

Walls, M. L., Whitbeck, L. B., Hoyt, D. R., \& Johnson, K. D. (2007). Early-onset alcohol use among Native American youth: Examining female caretaker influence. Journal of Marriage and Family, 69, 451-464. doi:10.1111/ j.1741-3737.2007.00376.x

Wei, M., Alvarez, A. N., Ku, T.-Y., Russell, D. W., \& Bonett, D. G. (2010). Development and validation of a Coping with Discrimination Scale: Factor structure, reliability, and validity. Journal of Counseling Psychology, 57, 328-344. doi:10.1037/a0019969

Wei, M., Heppner, P. P., Ku, T.-Y., \& Liao, K. Y.-H. (2010). Racial discrimination stress, coping, and depressive symptoms among Asian Americans: A moderation analysis. Asian American Journal of Psychology, 1, 136150. doi:10.1037/a0020157

Whitbeck, L. B., \& Armenta, B. E. (2015). Patterns of substance use initiation among Indigenous adolescents. Addictive Behaviors, 45, 172-179. doi:10.1016/j.addbeh. 2015.01.006

Whitbeck, L. B., Chen, X., Hoyt, D. R., \& Adams, G. W. (2004). Discrimination, historical loss and enculturation: Culturally specific risk and resiliency factors for alcohol abuse among American Indians. Journal of Studies on Alcohol, 65, 409-418. doi:10.15288/jsa.2004. 65.409

Whitbeck, L. B., Walls, M. L., \& Sittner Hartshorn, K. J. (2014). Indigenous adolescent development: Psychological, social and historical contexts. New York, NY: Psychology Press.

Whitesell, N. R., Kaufman, C. E., Keane, E. M., Crow, C. B., Shangreau, C., \& Mitchell, C. M. (2012). Patterns of substance use initiation among young adolescents in a Northern Plains American Indian tribe. American Journal of Drug and Alcohol Abuse, 38, 383-388. doi:10.3109/ 00952990.2012.694525

Williams, D. R., \& Mohammed, S. A. (2009). Discrimination and racial disparities in health: Evidence and needed research. Journal of Behavioral Medicine, 32, 2047. doi:10.1007/s10865-008-9185-0 